\title{
The Troll in Toll: Mal and Tram as bridges for TLR2 and TLR4 signaling
}

\author{
Frederick J. Sheedy ${ }^{1}$ and Luke A. J. O’Neill \\ School of Biochemistry and Immunology, Trinity College, Dublin, Ireland
}

\begin{abstract}
Signaling by two of the most important bacteria-sensing TLRs, TLR2 and TLR4, involves two adaptor proteins, MyD88 adaptor-like (Mal) and Toll/IL-1 receptor (TIR) domain-containing adaptor-inducing IFN- $\beta$ (Trif)-related adaptor molecule (TRAM). Recently, new insights into the functioning of these two adapters have emerged. Mal is required by both TLRs to act as a bridge to recruit the adaptor MyD88, leading ultimately to NF- $\kappa$ B activation. Similarly, TRAM acts as a bridge to recruit TRIF to the TLR4 complex, leading to activation of the transcription factor IFN regulatory factor 3. Consistent with Mal and TRAM being key points of control, recent evidence suggests that they are subject to regulation by phosphorylation. Further, a variant in Mal in humans has been found to protect against multiple infectious diseases. Finally, another TIR domain-containing adaptor, sterile $\alpha$ and HEAT/armadillo motif protein (SARM), has been shown to act as an inhibitor of TRIFdependent signaling. These recent discoveries add to the complexity of TLR signaling and highlight specific control mechanisms for TLR2 and TLR4 signaling. J. Leukoc. Biol. 82: 196-203; 2007.
\end{abstract}

Key Words: Toll-like receptor $\cdot$ signal transduction $\cdot$ TLR adapters · SARM · innate immunity · inflammation

\section{INTRODUCTION}

TLRs are one of the main groups of pathogen sensors in the innate immune response. They are a group of Class I transmembrane proteins, and 11 occur in humans and 13 in mice. They all sense evolutionarily conserved, microbial PAMPs [1]. TLR signaling ultimately results in the activation of transcription factors such as NF- $\mathrm{KB}$ and IFN regulatory factors (IRFs), which promote the transcription of proinflammatory cytokines and other proteins that promote host defense [2]. Much of the specificity used by the different TLR pathways is brought about by the use of different Toll-IL-1 receptor (IL-1R) (TIR) domain-containing adaptor proteins, of which there are currently five known in humans. They are MyD88, MyD88 adaptor-like (Mal; also known as TIRAP), TIR domain-containing adaptorinducing IFN- $\beta$ (TRIF), TRIF-related adaptor molecule (TRAM), and sterile $\alpha$ and HEAT/armadillo (ARM) motif protein (SARM) [3]. Upon activation, TLRs interact intracellularly with these adaptor proteins via TIR domains, and the adaptor proteins then recruit and activate downstream molecules such as IL-1R-associated kinases (IRAKs) and TNF-receptor-associated factor-6 (TRAF6), which then amplify the signal. The importance of TLR adapters in innate immunity is demonstrated by the recent discovery that the sea urchin, Strongylocentrotus purpuratis, which does not possess an adaptive immune system, has a predicted 222 TLRs and 26 TIR domaincontaining adapters [4].

In this review, we will focus on recent developments concerning Mal, TRAM, and SARM. Mal and TRAM have been shown to be bridging adapters. Mal recruits MyD88 to TLR2 and TLR4, and TRAM recruits TRIF to TLR4. The MyD88 pathway leads to activation of the transcription factors $N F-\kappa B$, IRF1, IRF5, and IRF7. The TRIF pathway leads to activation of IRF3. Mal and TRAM will govern these pathways and as might be expected, are subject to control by covalent modification, which will allow engagement with each pathway, the Trolls on the bridges, which need to be crossed to trigger signaling. We will also discuss recent findings in relation to the fifth adaptor, SARM, which has been shown to inhibit TRIF signaling.

\section{THE REPERTOIRE OF TIR DOMAIN- CONTAINING ADAPTERS}

The first TIR domain-containing adaptor to be discovered was MyD88. It was long known to have a role in signaling from the IL-1R [5] and was later discovered to associate with TLRs also [6]. Following the discovery that activation of NF-кB, p38 MAPK, and JNK was impaired in MyD88-deficient cells in response to all TLRs, except in the case of TLR3 [7], investigators sought additional TIR domain-containing adapters. Mal was discovered simultaneously by two groups [8,9] and was shown to drive NF- $\mathrm{B}$ activation. However Mal-deficient cells showed the same phenotype as MyD88-deficient cells in response to LPS, where delayed NF-кB activation was evident, and so it was postulated that Mal worked in conjunction with MyD88 on the TLR4 pathway. Activation of NF-кB and p38, however, is abolished in Mal-deficient cells in response to TLR2 ligands, and so it was realized that Mal is absolutely

\footnotetext{
${ }^{1}$ Correspondence: School of Biochemistry and Immunology, Trinity College, Dublin, Ireland. E-mail: sheedyf@tcd.ie

Received December 22, 2006; revised March 1, 2007; accepted March 20, 2007.

doi: $10.1189 / \mathrm{jlb} .1206750$
} 
required, in conjunction with MyD88, on the TLR2 pathway [10].

Following this, a third adaptor, TRIF, was discovered, which leads to late activation of NF-KB on the TLR3 and TLR4 but not TLR2 pathway [11], which explains the results found in MyD88 and Mal-deficient cells. The effect of TRIF here is to induce TNF- $\alpha$ production via activation of IRF3, with TNF- $\alpha$ acting in an autocrine manner to induce the late activation of NF-кB [12, 13]. TRIF, however, was found to be unable to interact with TLR4 [14], and subsequently, a fourth adaptor was found by bioinformatic searches of the databases, which had strong homology to the TIR domain of TRIF and was called TRAM [15] or TIR domain-containing adaptor molecule-2 [16]. This 235 amino acid protein contains a TIR domain in its C terminus, was found to interact with TLR4 and TRIF, and was theorized to act as a bridging adaptor in the MyD88-independent pathway in TLR4 signaling.

More recently, it has been discovered that the fifth TIR domain-containing adaptor, SARM, has a role in the TLR3 and TLR4 pathways in TRIF-dependent signaling [17]. SARM was first identified as a protein containing sterile $\alpha$ motifs (SAM) and ARM motifs [18]. Overexpression of human SARM, unlike the other adapters, did not lead to NF-кB activation [19]. The study by Carty et al. [17] demonstrated that overexpression of SARM blocked the induction of TRIF-dependent but not MyD88-dependent genes.

\section{CONTROLLING MAL AND TRAM MEMBRANE LOCALIZATION AND PHOSPHORYLATION}

Following the discovery of Mal, investigators initially thought it may function in a similar manner to MyD88 in TLR4 signaling. This activity was, however, later attributed to TRIF. The four adapters can now be thought of in two groups: the signaling adapters MyD88 and TRIF and the bridging adapters Mal and TRAM.

Human Mal (hMal) is a 25-kDa protein, 235 amino acids in length, which contains a TIR domain in its $\mathrm{C}$ terminus [8]. It was shown to interact with the TIR domains of TLR4 and TLR2 and also the TIR domain of MyD88 [20]. More recently, it has become clear that Mal serves as a bridging adaptor for TLR4 and MyD88, helping to recruit MyD88 to the plasma membrane, where it can activate downstream signaling components required to bring about the TLR4 response. Mal contains a phosphatidylinositol 4,5-bisphosphate (PIP2)-binding domain in its N-terminus, from amino acids 15 to 35 [21]. PIP2 binding to Mal functions to recruit Mal specifically to PIP2-rich regions in the plasma membrane. Blocking this localization abolishes TLR4-MyD88-dependent signaling. TLR2 and TLR4 are found in the plasma membrane, where PIP2 is enriched [22]. It was shown that Mal recruits MyD88 to the membrane via its TIR domain, and this is required for signaling from TLR4. A mutant construct, which places the PIP2-binding site of another protein, phospholipase $\mathrm{C}$, to the $\mathrm{C}$ terminus of MyD88 directs MyD88 to the membrane and restores LPS signaling in MyD88, Mal, and MyD88/Mal double-knockout cells. It would seem then that the sole function of Mal, at least in relation to NF-кB activation, is to recruit MyD88 to TLR4-rich regions in the plasma membrane. Perhaps other TLRs recruit MyD88 to distinct compartments via other, as-yet-unknown, mechanisms. It will be interesting to examine the requirement for $\mathrm{Mal}$ in recruiting MyD88 in TLR2 signaling and whether or not this process might be PIP2-dependent.

The results from the above study agree with a previous study, which modeled the TIR domains of Mal, MyD88, and TLR4 [20]. In this study, it was shown that the surface charge distribution of the TIR domains of TLR4 and MyD88 is largely electropositive and would therefore repel each other, whereas the surface charge distribution of the TIR domain of Mal is largely electronegative. Thus, Mal may serve to link both proteins in TLR4 signaling. Molecular models of the TIR domains of other TLRs may explain why Mal is not required to recruit MyD88.

The findings of the Kagan and Medzhitov study [21] not only serve to highlight what may be the main function of Mal but also provide insight into lipid metabolism and its regulation in TLR signaling. It was known that synthesis of PIP2 was regulated by $\beta 2$-integrins [23]. The only $\beta 2$-integrin expressed on macrophages is CD1lb [24]. The Kagan and Medzhitov study [21] demonstrated that CD11b-deficient cells are hyporesponsive to LPS. It was also shown that the localization of Mal to the plasma membrane by PIP2 binding is dependent on the $\beta 2$ integrin CD1lb in macrophages and also on the GTPase ADP ribosylation factor 6 (ARF6), which regulates production of PIP2 via activation of the enzyme PI-5K [25]. ARF6 is activated by integrins. Blocking the activity of ARF6 interferes with TLR4-MyD88-dependent signaling. It is possible then that the enzymes, which regulate the synthesis of PIP2, act as another level of control in TLR signaling. Perhaps their activity is up-regulated upon exposure to pathogens, which modulate a TLR response. These enzymes, however, represent another target for pathogens to manipulate the immune response. It is known that phosphatidylinositol metabolism is targeted widely by a number of microbial pathogens [26], including Salmonella typhimurium, which produces a phosphatase, SopB, interfering with PIP2 levels. This protein has been shown to interrupt Mal localization and therefore, disrupt TLR4 signaling [21].

Recently, Mal has been shown to undergo covalent modifications, which regulate its behavior. Gray et al. [27] demonstrated that Mal can be tyrosine-phosphorylated at residues 86 and 187 by Bruton's tyrosine kinase (Btk) following activation of TLR2 and TLR4. This phosphorylation is required for the activation of a pathway culminating in transactivation by the NF-кB subunit p65 via phosphorylation on serine 536. It was shown previously that Btk is required in TLR4 signaling to promote transactivation by p65 [28]. In addition to being tyrosine-phosphorylated, Mal is subsequently degraded following stimulation of TLR4 and TLR2 [29]. This degradation is mediated by suppressor of cytokine signaling-1 (SOCS-1), which itself is LPS-inducible [30]. SOCS-1 recognizes a proline, glutamic acid, serine, and threonine-rich area domain (PEST) in Mal, N-terminal to its TIR domain and subsequently, targets Mal for degradation by the addition of ubiquitin to lysine side-chains at amino acid positions 15 and 16. Ubiquitinated Mal is then degraded by the $26 \mathrm{~S}$ proteasome. Interfering with this process potentiates TLR signaling and particularly results in enhanced NF- $\mathrm{kB}$ activation and excessive 
cytokine responses. This SOCS-1-mediated degradation following stimulation of TLR2 and TLR4 requires tyrosine phosphorylation of Mal by Btk. As phosphorylated Mal functions to amplify the signal to NF- $\mathrm{B}$, targeting it for degradation is a good way to limit the TLR response.

Two other studies have provided additional insights into the regulation of Mal. Mansell and colleagues [31] suggested that Mal contains a TRAF6-binding site in its C terminus, from amino acids 188 to 193. It was shown that Mal can bind TRAF6 directly and that mutating this TRAF6-binding site creates a dominant-negative mutant of NF- $\kappa$ B activation and activation of MAPKs by TLR2 and TLR4. As MyD88 does not contain a putative TRAF6-binding site, unlike TRIF, which interacts directly with TRAF6 to activate NF-кB, the authors speculate that Mal binding to TRAF6 provides a MyD88independent means of inducing activation of MAPKs and NFкB. MyD88 does, however, bind IRAK-1, which contains a TRAF6-binding site, leading to NF- $\mathrm{B}$ activation. It is therefore possible that Mal acts in conjunction with MyD88 to potentiate the signal and cause increased levels of $\mathrm{NF}-\kappa \mathrm{B}$ activation through increased recruitment of TRAF6. This study was the first to suggest a distinct function for Mal independent of MyD88.

Another interesting feature of $\mathrm{Mal}$ is its recently discovered ability to interact with caspase-1 [32], which is an important inflammatory caspase, responsible for cleavage of pro-IL-1 $\beta$ into a catalytically active form secreted by cells. A yeast two-hybrid screen using Mal as bait isolated caspase-1 from a splenocyte library. The proteins were shown to interact endogenously. Mal was not required for caspase-1 activation. Instead, it was shown to be a substrate for caspase-1. Processing of Mal was shown to be required for it to activate NF-кB. This finding provides an important point of interaction between caspase-1-containing inflammasomes and TLRs. Caspase-1 must be activated in inflammasomes, which consist of another group of an innate pathogen sensor, nucleotide oligomerization domain-like receptors (NLRs), including the proteins Nalpl, Nalp3, and Ipaf. These proteins, similar to TLRs, respond to PAMPs, usually in the cytoplasmic environment [33]. As TLRs and NLRs may be engaged during infection, the ability of caspase-1 to activate Mal may be an important coordinating point between the two classes of innate receptors.

Figure 1 illustrates the domains and amino acids in Mal subject to control during signaling, and Figure $\mathbf{2}$ presents a Mal cycle to illustrate how Mal might signal during the TLR4 pathway. Although all of these discoveries have increased our knowledge of Mal, it is unclear yet whether these modifications occur solely to modulate its activity as a bridging adaptor or whether any novel MyD88-independent functions of Mal exist.

Regarding TRAM, we have less information about its biochemical regulation. However, there are clear parallels to Mal in terms of membrane localization and phosphorylation. Upon its discovery, TRAM was theorized to act as a bridging adaptor in the MyD88-independent pathway in TLR4 signaling. Recent studies have outlined how this occurs through biochemical modification similar to Mal and underline once again how control of the LPS response is tightly regulated. Rowe et al. [34] demonstrated that human TRAM contains a myristoylation site in its N-terminus from amino acids 1 to 8 . Addition of a myristoyl group to TRAM at this site directs TRAM to the plasma membrane, where it colocalizes with TLR4. Interfering with this myristoylation site abolishes MyD88-independent signaling from TLR4. It will be interesting to determine if myristoylation is dynamically regulated during LPS signaling.

Also similar to Mal, McGettrick and colleagues [35] demonstrated that TRAM is phosphorylated following LPS treatment. Mutational analysis revealed that membrane-associated TRAM is phosphorylated at serine 16. This phosphorylation is transient, appearing 15 min following exposure to LPS, peaking at $45 \mathrm{~min}$. However, phosphorylation of TRAM is followed, similar to Mal, by its apparent dissociation from the plasma membrane. It is tempting to speculate that TRAM, in this case,
Fig. 1. Sequence analysis of hMal and murine Mal (mMal). Protein sequences were downloaded from National Center for Biotechnology Information (NCBI) and aligned using Clustal W. Known domains and sites of interest are annotated. The PIP2-binding site is located from amino acids 15 to 35 . Lysines 15 and 16 are putative residues to which SOCS-1 attaches ubiquitin. The PEST domain is found between the PIP2binding site and the TIR domain. Tyrosines 86 and 187 are phosphorylated by Btk. Serine 180 is mutated to leucine in a relatively high frequency of the population, and heterozygosity at this locus offers protection to infectious disease. Mal also contains a TRAF6-binding site and a caspase-1 cleavage site in its $\mathrm{C}$ terminus.

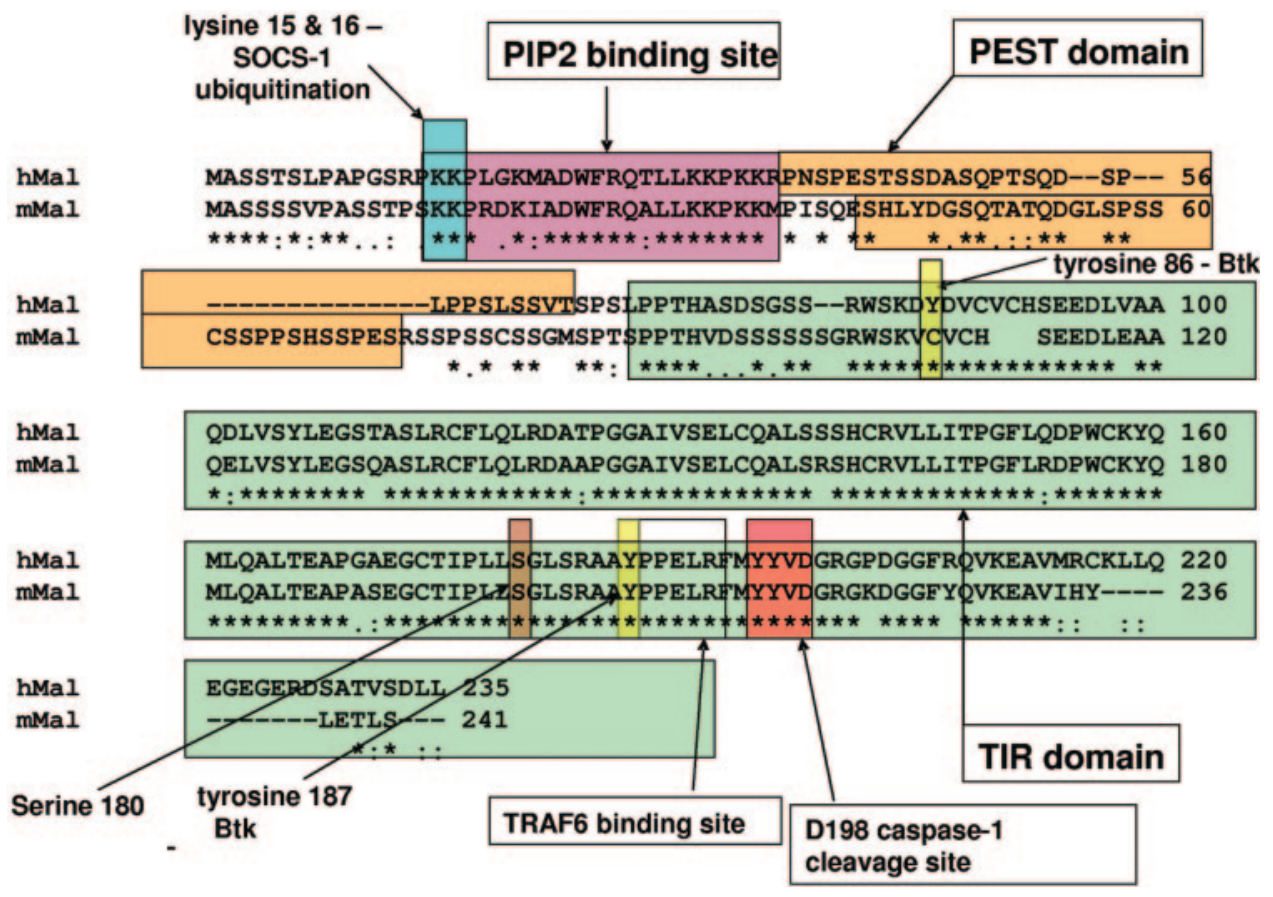

http://www.jleukbio.org 


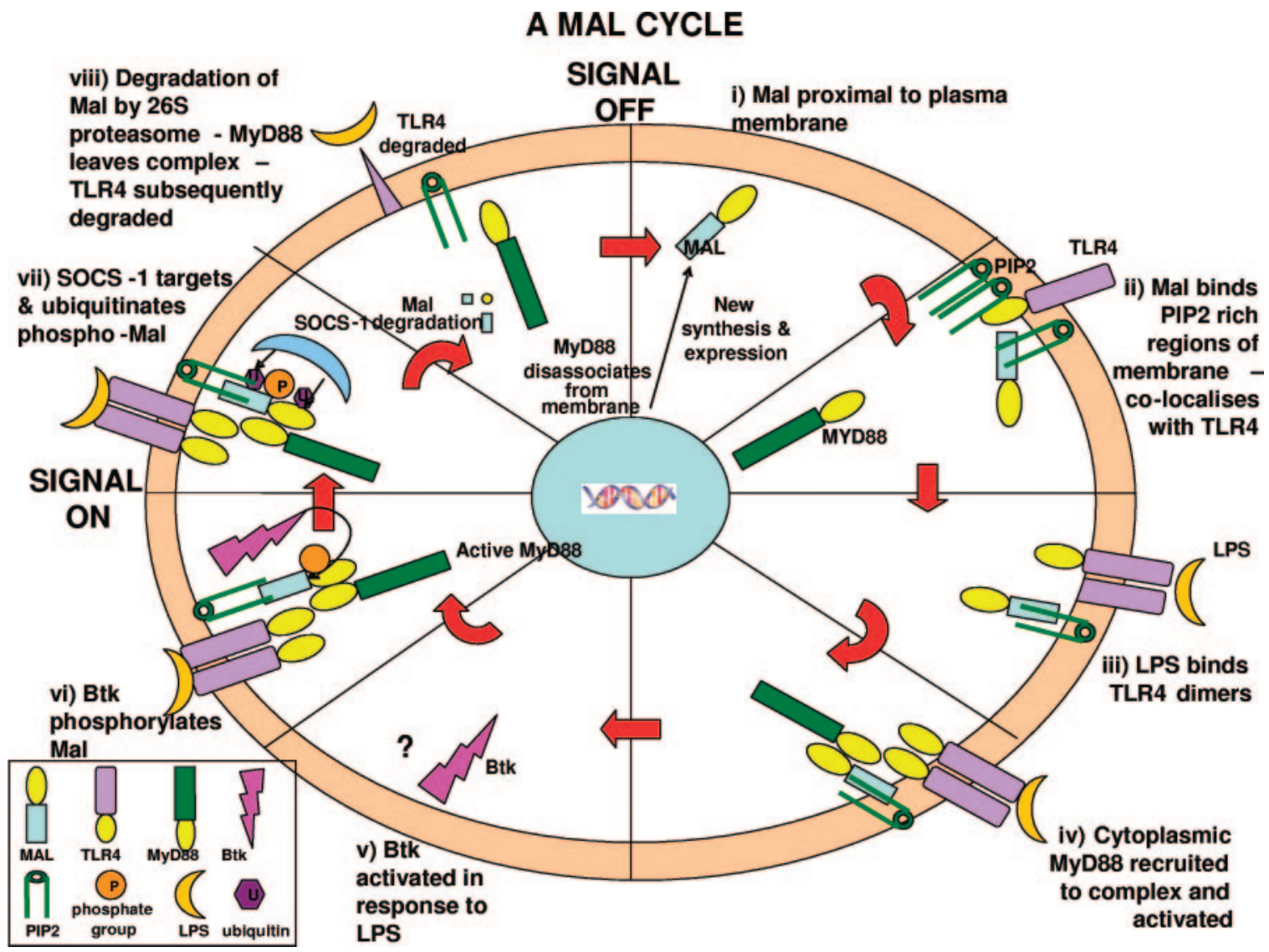

Fig. 2. Mal as a bridging adaptor in TLR4 signaling. i) Mal is synthesized and produced and ii) binds PIP2 in the plasma membrane via its PIP2-binding domain. It colocalizes with TLR4. iii) In cases of infection, LPS, derived from gram-negative bacteria, binds TLR4 dimers. iv) This leads to MyD88 recruitment to the Mal/TLR4 complex. MyD88 can then interact with downstream signaling molecules such as IRAK-4 and IRAK-1. These interact with further molecules, eventually resulting in the activation of NF-кB. v) By an as-yet-unknown mechanism, Btk is activated following LPS stimulation of the cell. vi) Btk phosphorylates Mal. The phosporylation of Mal is required for signaling. vii) Phospho-Mal is recognized by the ubiquitin ligase SOCS-1 (whose expression also increases in response to LPS). SOCS-1 uses its E3 ligase activity to attach ubiquitin residues to Mal. viii) This ubiquitination step targets Mal for degradation by the 26S proteasome. Mal is removed from the membrane. MyD88 can no longer interact directly with TLR4 and also leaves the membrane. This disrupts the signaling pathway and limits the response to LPS. Resynthesis of Mal will allow the cycle to continue.

is degraded similarly to Mal, in an effort to limit the LPS response. However, the phosphorylation event also seems to be critical in MyD88-independent LPS signaling, as inhibition of phosphorylation and overexpression of a S16A TRAM mutant inhibited activation of IRF3 by LPS. The kinase responsible for phosphorylation of TRAM was found to be protein kinase $\mathrm{C} \varepsilon$ (PKCE), which was known to have a role in LPS signaling [36], and its activation by other signaling molecules such as diacyglycerol [37] may again provide another level of control in the LPS response.

Analogous to Mal, therefore, the role of TRAM as a bridging adaptor in TLR4 signaling is now understood more clearly. A model for TRAM signaling is shown in Figure 3. It is unclear yet as to whether TRAM has any other functions, apart from its role as a bridging adaptor for TRIF in TLR4 signaling. The C-terminal TIR domain is highly conserved across species. Sequence similarity is also seen in the N-terminus of human, mouse, rat, cow, and dog TRAM, and the chimpanzee and macaque TRAM orthologs possess longer N-termini. The myristoylation site and serine 16 are absolutely conserved across the human, mouse, rat, cow, and dog. TRAM has a potential PEST domain located from amino acids 41 to 63 in murine, bovine, and canine TRAM. These features are illustrated in Figure 4A. Although the myristoylation site is not conserved in chimpanzee or macaque TRAM, analysis of their protein sequences by InterPro [38] revealed they may possess GOLD domains, which are commonly found in lipid or membraneassociated proteins and in this case, may function similarly to the myristoylation site to direct TRAM to the membrane. In addition, these TRAM orthologs possess signal peptides in their N-termini, which include a transmembrane region. These features are illustrated in Figure 4B.

Finally, TRIF-dependent signaling cannot only be manipulated by modification of TRAM but also by the action of SARM. As stated above, overexpression of SARM was shown to have an inhibitory effect on TRIF-dependent signaling [17]. The SARM protein interacts with TRIF, and this interaction requires its TIR domain. This interaction is weak in untreated cells but is enhanced by LPS or dsRNA treatment. SAM motifs are required for inhibition to occur. Whether inhibition occurs simply through sequestering of TRIF, preventing it from interacting with downstream signaling components, or whether SARM recruits other proteins to the TRIF-SARM complex via its SAM motifs is still unknown. It was also shown that treat- 


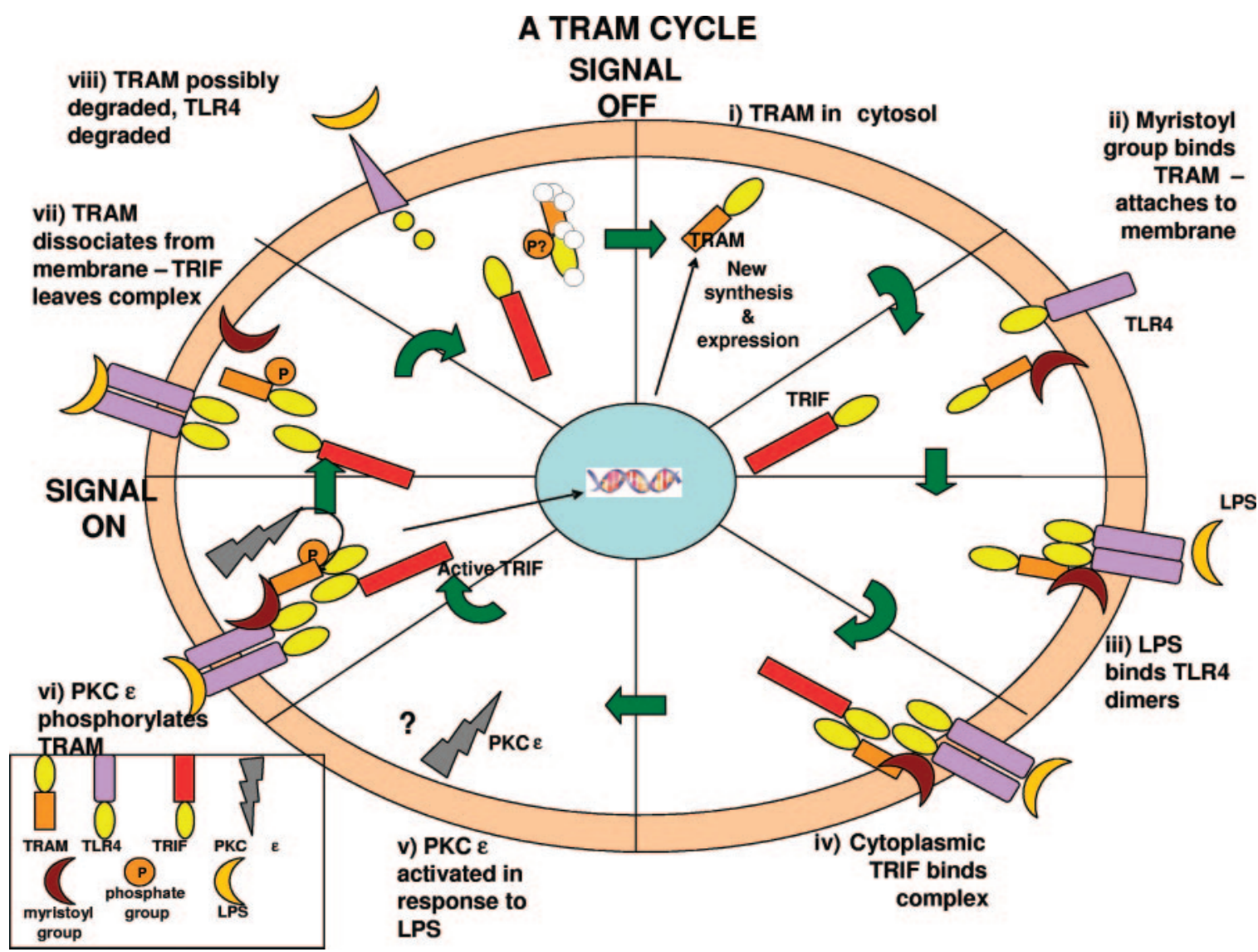

Fig. 3. TRAM as a bridging adaptor in TLR4 signaling. i) TRAM is produced in the cytoplasm. ii) TRAM is myristoylated in its N-terminus and becomes attached to the membrane in association with TLR4. iii) During infection, LPS, derived from gram-negative bacteria, binds TLR4 dimers. iv) This leads to the recruitment of TRIF to the TRAM/TLR4 complex. TRIF then interacts with downstream signaling molecules such as Tank-binding kinase-1, which phosphorylates and activates the transcription factor IRF3. TRIF also interacts with TRAF6 and receptor-interacting protein-1, which brings about the activation of NF- $\mathrm{kB}$. v) By an as-yet-unknown mechanism, PKCe is activated in response to LPS. vi) PKC $\varepsilon$ phosphorylates TRAM at serine 16. This phosphorylation event is required for signaling. vii) This phosphorylation event may also cause TRAM to leave the membrane. viii) With TRAM depleted from the membrane, TRIF no longer interacts with TLR4 and also leaves the membrane. This disrupts the signaling pathway and limits the response to LPS. TLR4 is also degraded at this point. Resynthesis of TRAM will allow the cycle to continue.

ment of cells with LPS enhances SARM expression, and it has been speculated that following TLR4 activation, SARM levels increase in cells and eventually bind TRIF to limit the LPSdriven response. In addition, deletion of the N-terminus of SARM enhances its inhibitory function but reduces its sensitivity to LPS-induced expression. SARM may be inherently unstable as a result of motifs in the N-terminus, which following LPS treatment, become more stable, allowing it to interact with TRIF to bring about its inhibitory function. Whether this ligand-induced enhancement of the SARM-TRIF interaction is a result of increased SARM expression or conformational changes in the SARM protein remains unclear.

\section{POLYMORPHISMS IN THE ADAPTERS}

A final, interesting, recent finding in relation to $\mathrm{Mal}$ is that genetic variation in the population may predispose individuals to infection. A study by Hawn et al. [39] genotyped Mal in a Vietnamese population in healthy control groups and in a population, which had suffered from tuberculosis (TB). They found four mutant alleles, two of which alter the amino acid sequence of Mal. One of these, the C539T or S180L allele, is associated with increased susceptibility to TB. The two variants, which do not alter Mal amino acid sequence, are also associated with increased susceptibility to TB. Blood was taken from patients homozygous for one of these alleles, C558T, and stimulated with TLR2 and TLR4 ligands. Levels of IL-6 were found to be decreased in these patients. It is unclear yet how these synonymous mutations lead to an altered phenotype, although it is likely that in the patients tested, the C558T allele is in linkage with some other functional mutation.

In another study, the frequency of multiple Mal mutations was examined in several populations in cases of pneumonia, malaria, bacteraemia, and TB [40]. It is striking that a particular variant, in which serine at position 180 is mutated to leucine, conferred protection in all of these diseases. In heterozygotes, the risk of disease is halved. Moreover, homozygotes for the leucine variant are absent in the African and Vietnamese populations, and they are present at low frequencies in the Caucasian populations. Biochemical evidence demonstrated that the leucine variant cannot reconstitute TLR2 signaling in Mal-deficient cells. The serine 180 residue is found in the TIR domain of Mal near an area-the DD loop. 
A

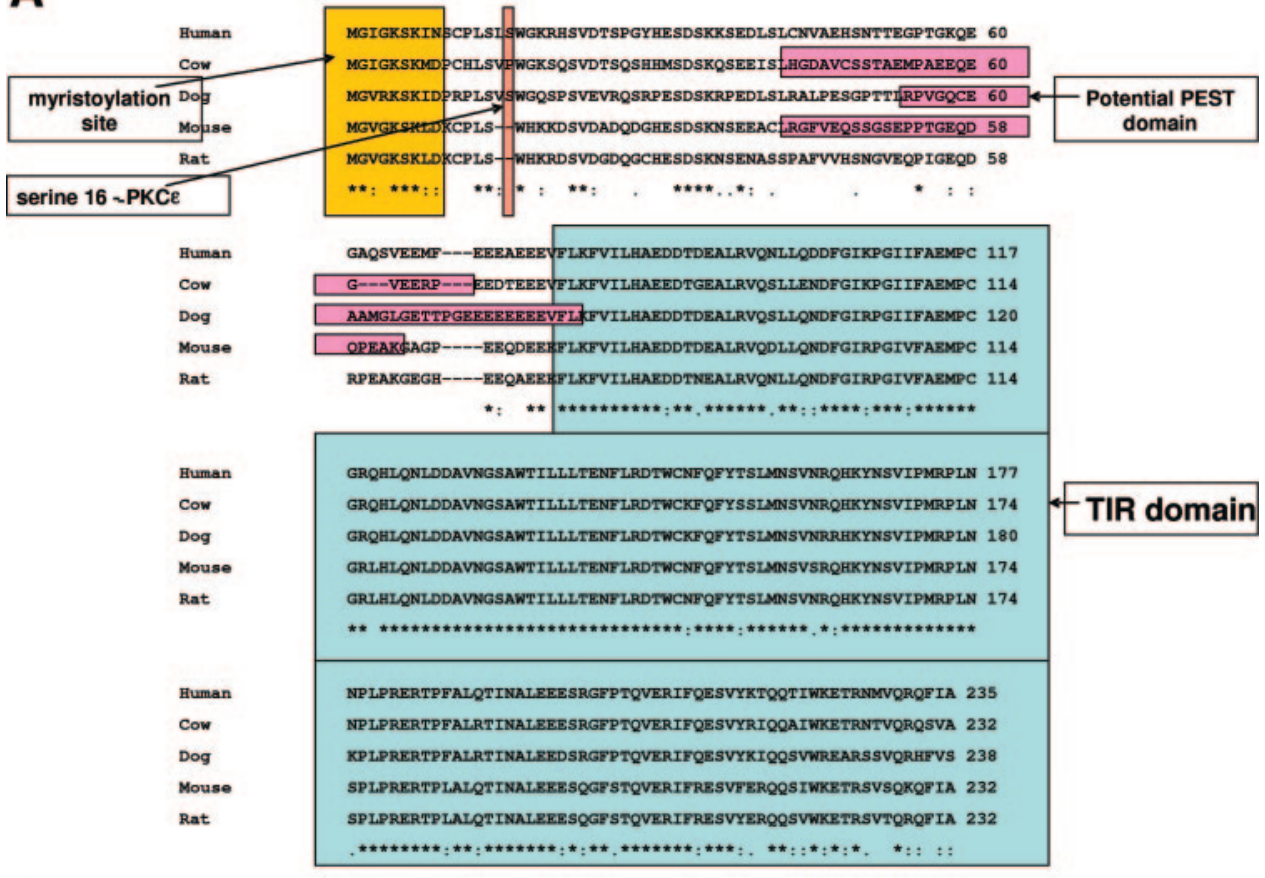

B

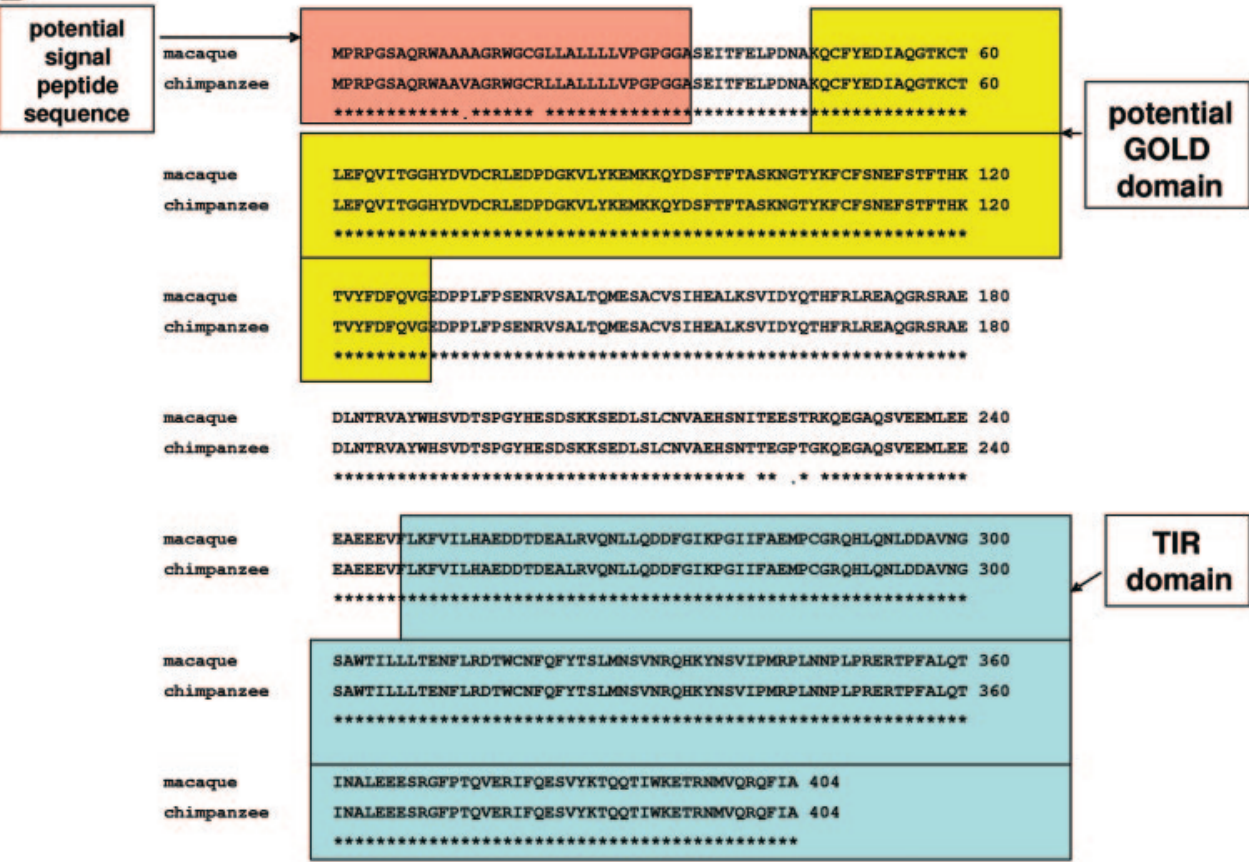

Fig. 4. (A) Amino acid sequence alignment of TRAM orthologs: human, Homo sapiens (Homo); cow, Bos taurus (Bos); dog, Canis familiaris (Canis); mouse, Mus musculus; and rat, Rattus norvegicus. Sequences were downloaded from NCBI and alignment performed using Clustal W. The myristoylation site is illustrated from amino acids 1 to 8. Potential PEST domains were discovered using the PESTfind analysis webtool, available at EMBnet Austria. Serine 16, which is phosphorylated by PKCe, is indicated. The highly conserved TIR domain is also shown in the $\mathrm{C}$ terminus. (B) Amino acid alignment of TRAM orthologs. Shown are TRAM from the macaque, Macaca mulatta, and the chimpanzee, Pan troglodytes. Sequence analysis was performed using the InterproScan sequence search tool, available at the European Bioinformatics Institute. A possible signal peptide sequence and GOLD domain are indicated. These are not found in other species' TRAM. These species do not possess a myristolylation site, and in this case, the GOLD domain may function in a similar manner to direct TRAM to the membrane.

The leucine 180 variant of Mal is unable to interact with TLR2. It would seem that the protective heterozygote state results in reduced signaling, whereas serine 180 homozygotes have an overactive, immune response, which is thought to render individuals more vulnerable to developing severe forms of malaria and bacterial disease. Leucine homozygotes, conversely, have little or no signaling and cannot mount an appropriate immune response. The low frequency of these in the populations studied adds weight to this theory. The attenuated signaling seen in the heterozygotes may therefore be the appropriate level of response required and may explain why it is advantageous and maintained in the population. Taken together, these results underline for the first time the importance of Mal in the innate response to infection in humans and validate TLR2 as a particularly important receptor for host defense in humans.

Analysis of the Single Nucleotide Polymorphism (SNP) database [41] reveals that the genes for the TIR domain-containing adapters are polymorphic. These data are presented in Table 1. It is significant that Mal seems to be the most polymorphic of the five adapters. This may be a result of the selective pressures associated with susceptibility to infection in different populations. This underlines the importance of Mal in the host response to infection in humans. There is only one reported polymorphism for TRAM in the human population reported in the SNP database, and it does not alter the amino acid sequence of the protein. In addition, one report suggests 
TABLE 1. Coding SNPs (cSNPs) in Genes for TLR Adapters, Which Alter Amino Acid Sequence of Protein

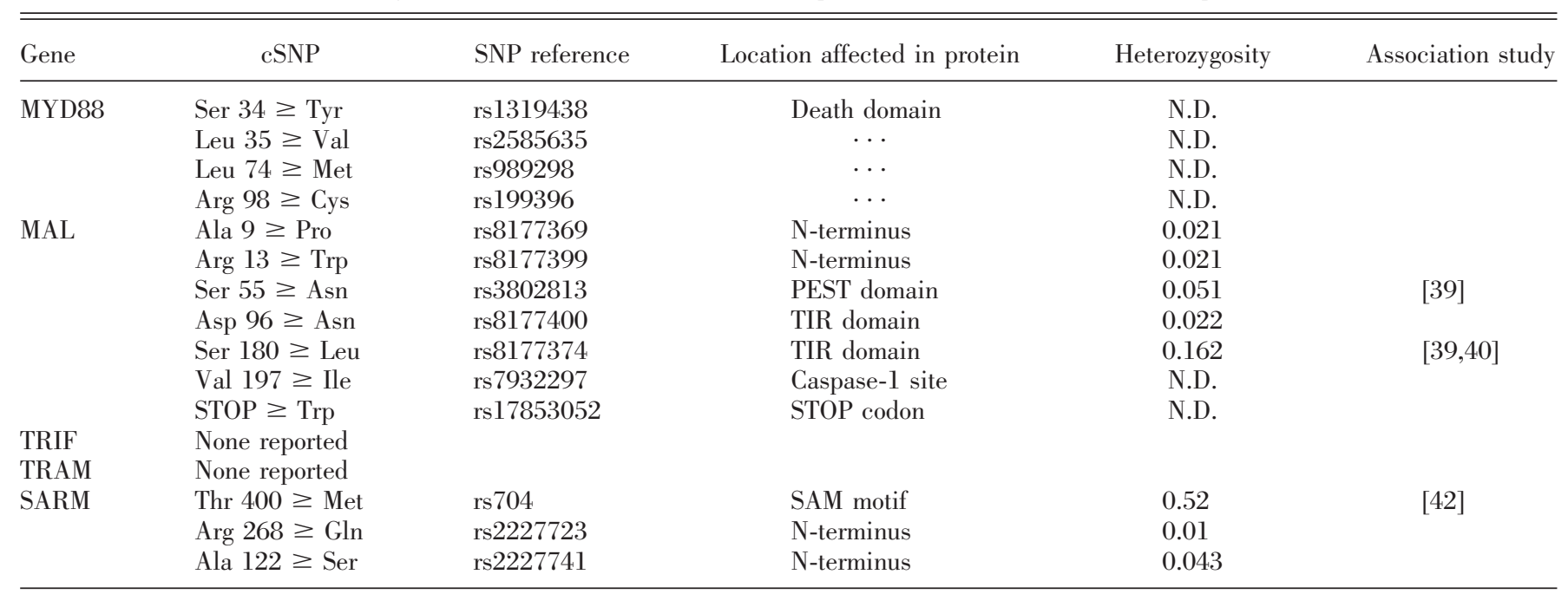

SNPs were downloaded from database SNP on NCBI. Only SNPs, which alter the coding sequence of the proteins, are listed here. N.D., Not determined.

that the SARM gene contains many SNPs, including mis-sense point mutations as well as insertions and deletions [42]. The SARM1 gene lies $600 \mathrm{Kbp}$ to the NO synthase 2A locus, which was shown in a previous study to be associated with TB susceptibility [43]. Whether this association is a result of linkage with polymorphisms in SARMI remains unclear.

\section{CONCLUSIONS}

Recent work on the adaptor proteins Mal, TRAM, and SARM has shown that the TLR4, TLR2, and TLR3 responses are tightly regulated by a variety of biochemical mechanisms. These controls highlight the importance of regulating TLR signaling, which if uncontrolled, can lead to disease. Several questions remain however.

Why does MyD88 require a bridging adaptor in the form of Mal for TLR2 and TLR4 signaling? It may be that Mal directs MyD88 to PIP2-rich regions, where TLR2 and TLR4 are also found. In addition, the question arises as to why TLR4 signaling via TRIF requires TRAM as a bridging adaptor, whereas TLR3 signaling, which occurs at endosomes, does not require TRIF. Again, subcellular localization may explain this. As stated before, another explanation for the requirement of bridges in TLR2 and TLR4 signaling may be the surface charge complementarity of the TIR domains involved, TLR2 and TLR4, which may be different from those of others in that they possess a positive surface charge, similar to MyD88 [20], and thus require adapters with negative surface charges in their TIR domains to link them to the signaling adapters. Further structural studies of the TIR domains of TRIF, TRAM, and TLR3 as well as other TLRs will be required to confirm this.

One advantage of using distinct adapters for TLR2 and TLR4 signaling is that these responses can be modulated easily by targeting these adapters without modifying the activity of the universal adaptor MyD88. In this way, TLR2 and TLR4 responses can be controlled without affecting other responses. Similarly, targeting TRAM as a limiting factor in TLR4 signaling provides a method to control TLR4 signaling without affecting TLR3 signaling, which requires TRIF solely. Similarly, use of SARM as a negative inhibitor of TLR3 and TLR4 signaling provides a unique method to limit these responses without affecting other TLR responses. It can then be said that not only does differential adaptor use lead to specificity in the TLR response but also leads to specific control of each pathway.

\section{ACKNOWLEDGMENTS}

Science Foundation Ireland, Health Research Board of Ireland, and Irish Research Council for Science, Engineering and Technology are gratefully acknowledged for providing financial support to the authors.

\section{REFERENCES}

1. Janeway Jr., C. A. (1989) Approaching the asymptote? Evolution and revolution in immunology. Cold Spring Harb. Symp. Quant. Biol. 54 (Pt. 1), 1-13.

2. Miggin, S. M., O'Neill, L. A. (2006) New insights into the regulation of TLR signaling. J. Leukoc. Biol. 80, 220-226.

3. McGettrick, A. F., O'Neill, L. A. (2004) The expanding family of MyD88like adaptors in Toll-like receptor signal transduction. Mol. Immunol. 41, $577-582$.

4. Rast, J. P., Smith, L. C., Loza-Coll, M., Hibino, T., Litman, G. W. (2006) Genomic insights into the immune system of the sea urchin. Science 314, 952-956.

5. Wesche, H., Henzel, W. J., Shillinglaw, W., Li, S., Cao, Z. (1997) MyD88: an adapter that recruits IRAK to the IL-1 receptor complex. Immunity 7 , 837-847.

6. Kawai, T., Adachi, O., Ogawa, T., Takeda, K., Akira, S. (1999) Unresponsiveness of MyD88-deficient mice to endotoxin. Immunity 11, 115-122.

7. Jiang, Z., Zamanian-Daryoush, M., Nie, H., Silva, A. M., Williams, B. R., Li, X. (2003) Poly(I-C)-induced Toll-like receptor 3 (TLR3)-mediated activation of NFK B and MAP kinase is through an interleukin-1 receptorassociated kinase (IRAK)-independent pathway employing the signaling components TLR3-TRAF6-TAK1-TAB2-PKR. J. Biol. Chem. 278, 16713-16719.

8. Fitzgerald, K. A., Palsson-McDermott, E. M., Bowie, A. G., Jefferies, C. A., Mansell, A. S., Brady, G., Brint, E., Dunne, A., Gray, P., Harte, M. T., McMurray, D., Smith, D. E., Sims, J. E., Bird, T. A., O’Neill, L. A. 
(2001) Mal (MyD88-adapter-like) is required for Toll-like receptor-4 signal transduction. Nature 413, 78-83.

9. Horng, T., Barton, G. M., Medzhitov, R. (2001) TIRAP: an adapter molecule in the Toll signaling pathway. Nat. Immunol. 2, 835-841.

10. Yamamoto, M., Sato, S., Hemmi, H., Sanjo, H., Uematsu, S., Kaisho, T., Hoshino, K., Takeuchi, O., Kobayashi, M., Fujita, T., Takeda, K., Akira, S. (2002) Essential role for TIRAP in activation of the signaling cascade shared by TLR2 and TLR4. Nature 420, 324-329.

11. Yamamoto, M., Sato, S., Hemmi, H., Hoshino, K., Kaisho, T., Sanjo, H., Takeuchi, O., Sugiyama, M., Okabe, M., Takeda, K., Akira, S. (2003) Role of adaptor TRIF in the MyD88-independent Toll-like receptor signaling pathway. Science 301, 640-643.

12. Covert, M. W., Leung, T. H., Gaston, J. E., Baltimore, D. (2005) Achieving stability of lipopolysaccharide-induced NF-кB activation. Science $\mathbf{3 0 9}$, 1854-1857.

13. Yamamoto, M., Sato, S., Mori, K., Hoshino, K., Takeuchi, O., Takeda, K., Akira, S. (2002) Cutting edge: a novel Toll/IL-1 receptor domain-containing adapter that preferentially activates the IFN- $\beta$ promoter in the Tolllike receptor signaling. J. Immunol. 169, 6668-6672.

14. Oshiumi, H., Matsumoto, M., Funami, K., Akazawa, T., Seya, T. (2003) TICAM-1, an adaptor molecule that participates in Toll-like receptor 3 -mediated interferon- $\beta$ induction. Nat. Immunol. 4, 161-167.

15. Yamamoto, M., Sato, S., Hemmi, H., Uematsu, S., Hoshino, K., Kaisho, T., Takeuchi, O., Takeda, K., Akira, S. (2003) TRAM is specifically involved in the Toll-like receptor 4-mediated MyD88-independent signaling pathway. Nat. Immunol. 4, 1144-1150.

16. Oshiumi, H., Sasai, M., Shida, K., Fujita, T., Matsumoto, M., Seya, T. (2003) TIR-containing adapter molecule (TICAM)-2, a bridging adapter recruiting to Toll-like receptor 4 TICAM-1 that induces interferon- $\beta$. J. Biol. Chem. 278, 49751-49762.

17. Carty, M., Goodbody, R., Schroder, M., Stack, J., Moynagh, P. N., Bowie, A. G. (2006) The human adaptor SARM negatively regulates adaptor protein TRIF-dependent Toll-like receptor signaling. Nat. Immunol. 7, 1074-1081.

18. Mink, M., Fogelgren, B., Olszewski, K., Maroy, P., Csiszar, K. (2001) A novel human gene (SARM) at chromosome $17 \mathrm{q} 11$ encodes a protein with a SAM motif and structural similarity to armadillo/ $\beta$-catenin that is conserved in mouse, Drosophila, and Caenorhabditis elegans. Genomics 74, 234-244.

19. Liberati, N. T., Fitzgerald, K. A., Kim, D. H., Feinbaum, R., Golenbock, D. T., Ausubel, F. M. (2004) Requirement for a conserved Toll/interleukin-1 resistance domain protein in the Caenorhabditis elegans immune response. Proc. Natl. Acad. Sci. USA 101, 6593-6598.

20. Dunne, A., Ejdeback, M., Ludidi, P. L., O’Neill, L. A., Gay, N. J. (2003) Structural complementarity of Toll/interleukin-1 receptor domains in Tolllike receptors and the adaptors Mal and MyD88. J. Biol. Chem. 278, 41443-41451.

21. Kagan, J. C., Medzhitov, R. (2006) Phosphoinositide-mediated adaptor recruitment controls Toll-like receptor signaling. Cell 125, 943-955.

22. McLaughlin, S., Wang, J., Gambhir, A., Murray, D. (2002) PIP(2) and proteins: interactions, organization, and information flow. Annu. Rev. Biophys. Biomol. Struct. 31, 151-175.

23. Hynes, R. O. (2002) Integrins: bidirectional, allosteric signaling machines. Cell 110, 673-687.

24. Bhat, N., Perera, P. Y., Carboni, J. M., Blanco, J., Golenbock, D. T., Mayadas, T. N., Vogel, S. N. (1999) Use of a photoactivatable taxol analogue to identify unique cellular targets in murine macrophages: identification of murine CD18 as a major taxol-binding protein and a role for Mac-1 in taxol-induced gene expression. J. Immunol. 162, 7335-7342.

25. Honda, A., Nogami, M., Yokozeki, T., Yamazaki, M., Nakamura, H., Watanabe, H., Kawamoto, K., Nakayama, K., Morris, A. J., Frohman, M. A., Kanaho, Y. (1999) Phosphatidylinositol 4-phosphate 5-kinase $\alpha$ is a downstream effector of the small $G$ protein ARF6 in membrane ruffle formation. Cell 99, 521-532.
26. Pizarro-Cerda, J., Cossart, P. (2004) Subversion of phosphoinositide metabolism by intracellular bacterial pathogens. Nat. Cell Biol. 6, 10261033.

27. Gray, P., Dunne, A., Brikos, C., Jefferies, C. A., Doyle, S. L., O'Neill, L. A (2006) MyD88 adapter-like (Mal) is phosphorylated by Bruton's tyrosine kinase during TLR2 and TLR4 signal transduction. J. Biol. Chem. 281, 10489-10495.

28. Doyle, S. L., Jefferies, C. A., O’Neill, L. A. (2005) Bruton's tyrosine kinase is involved in p65-mediated transactivation and phosphorylation of p65 on serine 536 during NFкB activation by lipopolysaccharide. J. Biol. Chem. 280, 23496-23501.

29. Mansell, A., Smith, R., Doyle, S. L., Gray, P., Fenner, J. E., Crack, P. J., Nicholson, S. E., Hilton, D. J., O'Neill, L. A., Hertzog, P. J. (2006) Suppressor of cytokine signaling 1 negatively regulates Toll-like receptor signaling by mediating Mal degradation. Nat. Immunol. 7, 148-155.

30. Baetz, A., Frey, M., Heeg, K., Dalpke, A. H. (2004) Suppressor of cytokine signaling (SOCS) proteins indirectly regulate Toll-like receptor signaling in innate immune cells. J. Biol. Chem. 279, 54708-54715.

31. Mansell, A., Brint, E., Gould, J. A., O’Neill, L. A., Hertzog, P. J. (2004) Mal interacts with tumor necrosis factor receptor-associated factor (TRAF)-6 to mediate NF-кB activation by Toll-like receptor (TLR)-2 and TLR4. J. Biol. Chem. 279, 37227-37230.

32. Miggin, S. M., Palsson-McDermott, E., Dunne, A., Jefferies, C., Pinteaux, E., Banahan, K., Murphy, C., Moynagh, P., Yamamoto, M., Akira, S., Rothwell, N., Golenbock, D., Fitzgerald, K. A., O'Neill, L. A. (2007) $\mathrm{NF}-\{\kappa\} \mathrm{B}$ activation by the Toll-IL-1 receptor domain protein MyD88 adapter-like is regulated by caspase-1. Proc. Natl. Acad. Sci. USA 104, 3372-3377.

33. Creagh, E. M., O'Neill, L. A. (2006) TLRs, NLRs and RLRs: a trinity of pathogen sensors that co-operate in innate immunity. Trends Immunol. 27, 352-357.

34. Rowe, D. C., McGettrick, A. F., Latz, E., Monks, B. G., Gay, N. J., Yamamoto, M., Akira, S., O’Neill, L. A., Fitzgerald, K. A., Golenbock, D. T. (2006) The myristoylation of TRIF-related adaptor molecule is essential for Toll-like receptor 4 signal transduction. Proc. Natl. Acad. Sci. USA 103, 6299-6304.

35. McGettrick, A. F., Brint, E. K., Palsson-McDermott, E. M., Rowe, D. C., Golenbock, D. T., Gay, N. J., Fitzgerald, K. A., O'Neill, L. A. (2006) Trif-related adapter molecule is phosphorylated by PKC $\{\varepsilon\}$ during Tolllike receptor 4 signaling. Proc. Natl. Acad. Sci. USA 103, 9196-9201.

36. Castrillo, A., Pennington, D. J., Otto, F., Parker, P. J., Owen, M. J., Bosca, L. (2001) Protein kinase $\mathrm{C} \varepsilon$ is required for macrophage activation and defense against bacterial infection. J. Exp. Med. 194, 1231-1242.

37. Mellor, H., Parker, P. J. (1998) The extended protein kinase C superfamily. Biochem. J. 332, 281-292.

38. InterPro Database, European Molecular Biology Laboratory-European Bioinformatics Institute, www.ebi.ac.uk/InterProScan.

39. Hawn, T. R., Dunstan, S. J., Thwaites, G. E., Simmons, C. P., Thuong, N. T., Lan, N. T., Quy, H. T., Chau, T. T., Hieu, N. T., Rodrigues, S., Janer, M., Zhao, L. P., Hien, T. T., Farrar, J. J., Aderem, A. (2006) A polymorphism in Toll-interleukin 1 receptor domain containing adaptor protein is associated with susceptibility to meningeal tuberculosis. J. In fect. Dis. 194, 1127-1134.

40. Khor, C. C., Chapman, S. J., Vannberg, F. O., Dunne, A., Murphy, C., Ling, E. Y., Frodsham, A. J., Walley, A. J., Kyrieleis, O., Khan, A., et al. (2007) A Mal functional variant is associated with protection against invasive pneumococcal disease, bacteremia, malaria and tuberculosis. Nat. Genet. 39, 523-528.

41. Database, Single Nucleotide Polymorphisms, National Center for Biotechnology Information, www.ncbi.nlm.nih.gov/projects/SNP.

42. Mink, M., Csiszar, K. (2005) SARM1: A candidate gene in the onset of hereditary infectious/inflammatory diseases. Clin. Immunol. 115, 333334.

43. Frodsham, A. J., Hill, A. V. (2004) Genetics of infectious diseases. Hum. Mol. Genet. 13, R187-R194. 